\title{
Dessolventização e Tostagem do Farelo de Soja: Simulação e Análise Utilizando o HYSYS ${ }^{\circledR}$
}

\author{
H. D. S. SALINAS ${ }^{1}$, F.R.G.B. SILVA' ${ }^{1}$, L. M. M. JORGE ${ }^{1}$, P. R. PARAÍSO ${ }^{1}$ \\ ${ }^{1}$ Universidade Estadual de Maringá, Departamento de Engenharia Química \\ E-mail para contato: paulo@uem.br
}

\begin{abstract}
RESUMO -A separação do hexano, em mistura com o farelo de soja, é realizada normalmente em um equipamento denominado dessolventizador/tostador (DT). A operação industrial é denominada de dessolventização/tostagem, sendo extremamente necessária para a obtenção de farelo de soja com qualidade, e que atenda as especificações do mercado. No entanto, esta é uma das operações que apresentam um dos maiores consumos de energia nas indústrias de processamento de soja. Desta forma, o principal objetivo do presente trabalho é estudar o comportamento das variáveis envolvidas no processo, com o intuito de recuperar o máximo de hexano, com a menor quantidade de energia possível. Para tanto, foram realizadas simulações do DT, em regime estacionário, utilizando o software HYSYS $^{\circledR}$ a fim de verificar o comportamento operacional de variáveis importantes do processo, tais como temperatura e vazão de alimentação de farelo de soja. Os resultados obtidos mostram que novos pacotes de equilíbrio contidos no software precisam ser testados, e novos ajustes do modelo precisam ser efetuados, no entanto apontam que o software HYSYS pode ser usado como ferramenta para otimizar o processo de dessolventização e tostagem do farelo de soja.
\end{abstract}

\section{INTRODUÇÃO}

A soja é uma importante oleaginosa mundial, tendo como produtos de sua extração o óleo de soja e farelo de soja, sendo o último considerado padrão como fonte de proteína, e amplamente utilizado em todo o mundo na fabricação de ração animal, devido a sua palatabilidade, digestibilidade e melhoria da conversão alimentar. O Brasil se destaca como um dos maiores produtores mundiais de soja juntamente com os Estados Unidos e a Argentina. De acordo com o levantamento realizado pela (United States Department of Agriculture) o Brasil terá uma safra 2014/2015 recorde alcançando a produção de 95,5 milhões de toneladas, ficando somente atrás dos EUA com 108,0 milhões de toneladas na safra 2014/2015. Segundo a ABIOVE (Associação Brasileira das Industrias de Óleos Vegetais) só no ano de 2014 a produção do farelo de soja no Brasil alcançou 28,0 milhões de toneladas, tal produção vem crescendo ano após ano e a estimativa para o ano de 2015 é de 29,3 milhões de toneladas.

Sabendo da importância dessa oleaginosa bem como os seus subprodutos, diversos estudos vem sendo realizados a fim de proporcionar uma melhor eficiência nos processos de extração, bem como na melhoria da qualidade desses produtos. Segundo Dahlen \& Lindh 
(1983) e Schumacher (1983), o processo de dessolventização/tostagem do farelo de soja é a parte do processo responsável pela maior perda de solvente no processo de extração do óleo de soja. E o desempenho do DT define a quantidade remanescente de hexano no farelo ao final do processo o que possui grande importância, tendo em vista a contaminação do meio ambiente, segurança industrial, qualidade do farelo de soja e economia do processo.

Ainda sobre esta etapa do processo, conforme Paraíso (2001), o DT opera continuamente no meio industrial e em duas etapas. Sendo que na primeira ocorre a dessolvetização, onde o vapor direto e superaquecido entra em contato com a torta visando à separação da maior parte do hexano que ficou retido durante a operação de extração. $\mathrm{Na}$ segunda etapa ocorre a tostagem onde o vapor saturado entra em contato com o farelo de forma indireta, permitindo a evaporação do restante de hexano e promovendo um tratamento térmico, a fim de, destruir algumas enzimas prejudiciais a digestibilidade.

Para que melhorias sejam alcançadas, o estudo das mudanças nas variáveis do processo vem sendo realizado experimentalmente por diversos autores, através de trabalhos em laboratórios e plantas em escala piloto com a utilização de diferentes composições do farelo de soja (Cardarelli \& Crapiste, 1996; Grant, Eager, Pepper, \& Mathews, 1983; Mustakas, Moulton, Baker, \& Kwolek, 1981; Wolff, 1983; Paraíso, 2001).

Uma das formas de obter essas melhorias é por meio de simulações computacionais e, atualmente, existem boas alternativas para o uso de ferramentas computacionais na simulação de processos. Uma delas é a utilização do simulador HYSYS ${ }^{\circledR}$ que, de acordo com Meneguelo (2007), é um simulador bastante utilizado em projetos e análise de processos da indústria química devido ao caráter intuitivo do simulador na sua utilização e, além disso, as etapas para a construção de um caso são apresentadas de uma forma bastante simples e objetiva.

Desta forma, o objetivo do presente trabalho foi desenvolver um modelo para o dessolventizador/tostador, utilizando as diferentes operações disponíveis no software HYSYS $^{\circledR}$, e comparar os resultados obtidos com dados de um processo real, tais como da temperatura do farelo ao longo de cada estágio bem como a retirada de hexano presente na torta com a variação da alimentação de vapor na planta

\section{MODELO}

O processo de dessolventização e tostagem do farelo de soja foi modelado empregando-se o software HYSYS ${ }^{\circledR}$. No entanto, uma vez que o mesmo não dispõe de um banco de dados específico para o farelo de soja, a celulose foi escolhida para adequadamente realizar as simulações computacionais do processo, uma vez que este composto possui propriedades químicas e físicas semelhantes às do farelo de soja, bem como as propriedades críticas de temperatura e pressão. A adição da celulose à lista de constituintes do HYSYS ${ }^{\circledR}$ pode ser realizada utilizando-se a teoria da contribuição de grupos do método UNIFAC, no qual é possível criar um componente hipotético a partir de sua fórmula estrutural e quantificando os grupos básicos formadores da molécula. Uma outra forma de se adicionar a celulose à lista de componentes do $\mathrm{HYSYS}^{\circledR}$, é utilizar a glicose, monômero formador da celulose, que já pode ser encontrada no banco de dados do software em questão, facilitando, desta forma, a inserção dos componentes, levando-se em conta que todas as propriedades 
físicas e químicas já estão caracterizadas, além de fornecer coeficientes binários mais precisos quando em contato com outros componentes.

O pacote de propriedades empregado para realizar as simulações foi o NRTL-Ideal, que leva em conta as atividades dos componentes, com as frações molares dos mesmos fornecendo valores de propriedades mais corretos, e também tem sido amplamente empregado em simulações de processos que envolvem o equilíbrio de fases.

O software HYSYS ${ }^{\circledR}$ normalmente é empregado para simular processos de separação de hidrocarbonetos. Desta forma, o mesmo não possui uma operação de dessolventização e tostagem pronta para utilização, sendo então necessário combinar algumas operações para a construção de um dessolventizador/tostador. Nos estágios de dessolventização, existe o contato direto do vapor de água com a torta - farelo de soja mais hexano - e, desta forma, para modelar as etapas de dessolventização foi empregada a operação existente no HYSYS ${ }^{\circledR}$ chamada de absorber, modelada possuindo dois estágios, cada um deles possuindo um absorber. Por outro lado, nas etapas de tostagem, a torta não entra em contato direto com o vapor de água, e assim, utilizou-se para o modelo um aquecedor e um separador flash em cada estágio. A modelagem desta etapa do processo foi realizada considerando-se três estágios. Um fluxograma do processo de dessolventização e tostagem desenvolvido a partir das operações existentes no HYSYS ${ }^{\circledR}$ pode ser visto na Figura 1.

Figura 1 - Fluxograma do processo de Dessolventização/Tostagem

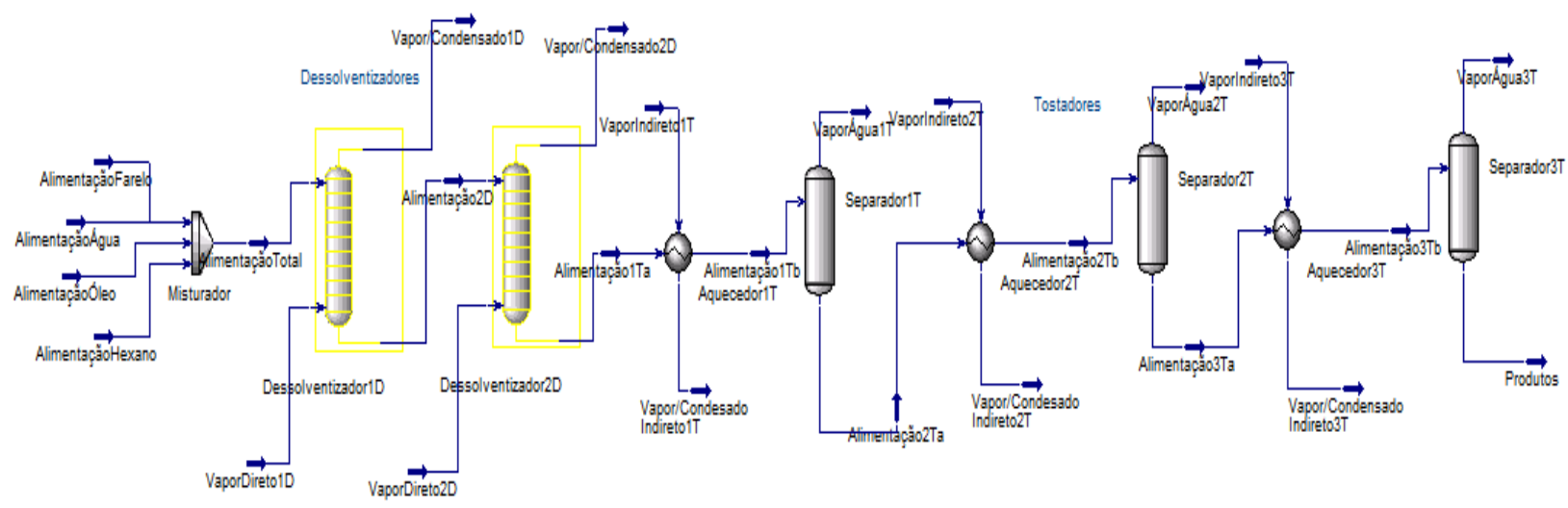

\section{RESULTADOS}

Para avaliar a eficiência do modelo desenvolvido, dados de temperatura do farelo, quantidade de hexano presente no farelo de soja na saída do dessolventizador/tostador, umidade do farelo de soja e vazão de alimentação de farelo, obtidos por meio de uma simulação, foram comparados com dados fornecidos por uma indústria de processamento de soja do noroeste do Paraná obtidos em Paraíso (2001), bem como com dados fornecidos pelas simulações do autor. Um comparativo entre estes dados pode ser visualizado na Tabela 1 abaixo, sendo possível observar que os dados obtidos por meio da simulação do modelo desenvolvido apresentam uma boa concordância com os dados da indústria e aqueles apresentados em Paraíso (2001). 
Tabela 1- Comparação entre os dados obtidos por meio da simulação no HYSYS ${ }^{\circledR}$, da indústria de processamento e fornecidos em Paraíso (2001).

\begin{tabular}{c|c|c|c|c|c}
\hline Variáveis & Indústria & \multicolumn{2}{|c|}{ Simulação Paraíso } & \multicolumn{2}{c}{ Simulação HYSYS } \\
\hline- & Dados & Dados & Desvio\% & Dados & Desvio\% \\
\hline $\begin{array}{c}\text { Temperatur } \\
\text { a }\end{array}$ & $118^{\circ} \mathrm{C}$ & $116,7^{\circ} \mathrm{C}$ & $1,10 \%$ & $105,7^{\circ} \mathrm{C}$ & $10,42 \%$ \\
\hline Hexano & $280 \mathrm{ppm}$ & $262 \mathrm{ppm}$ & $6,43 \%$ & $0 \mathrm{ppm}$ & - \\
\hline Umidade & $19,0 \%$ & $18,9 \%$ & $0,53 \%$ & $19,51 \%$ & $2,68 \%$ \\
\hline Vazão & $35530,0 \mathrm{~kg} / \mathrm{h}$ & $35394,5 \mathrm{~kg} / \mathrm{h}$ & $0,38 \%$ & $35640 \mathrm{~kg} / \mathrm{h}$ & $0,31 \%$ \\
\hline
\end{tabular}

Em decorrência dos resultados apresentados na Tabela 1, comprovando a eficiência do modelo desenvolvido, o mesmo foi estendido de forma a possuir não somente três, mas cinco estágios na operação de tostagem do farelo de soja. Na sequência, novas simulações foram realizadas, para diferentes condições de operação, com o intuito de avaliar o comportamento do dessolventizador/tostador. Desta forma, dados de temperatura nos estágios de dessolventização e tostagem obtidos foram comparados com os dados da indústria fornecidos em Paraíso et al. (2007), como pode ser observado na Figura 2.

Figura 2 - Perfil de temperatura ao longo do dessolventizador/tostador

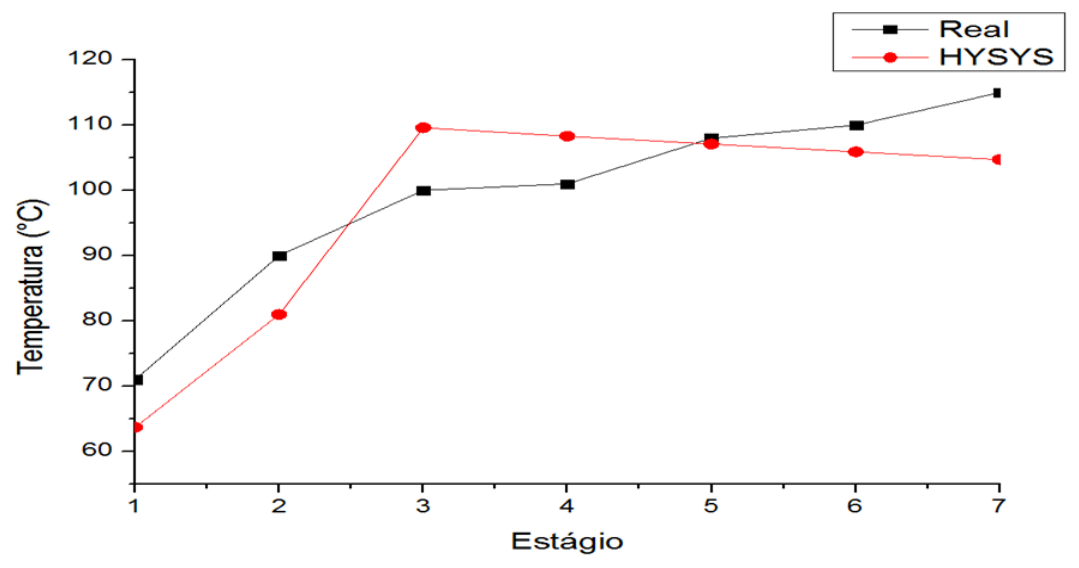

Na Figura 2, é possível observar claramente que a temperatura decresce ao longo dos estágios logo após a passagem pelo terceiro estágio, ao contrário do que ocorre nos processos reais. A Tabela 2 apresenta o comparativo destes dados com o respectivo desvio. Apesar destes desvios apresentarem baixos valores, que poderiam ser considerados aceitáveis como resultado, o comportamento da temperatura no processo simulado diverge do comportamento esperado. Desta forma, pode-se afirmar que o modelo de equilíbrio utilizado, o NRTL - Ideal, pode não ser apropriado para representar adequadamente os estágios de equilíbrio, que representam cada um dos estágios de um dessolventizador/tostador real, mostrando a necessidade de se investigar outros modelos que fazem parte do pacote do HYSYS ${ }^{\circledR}$, bem como efetuar ajustes nos modelos das operações empregadas para representar $o$ dessolventizador/tostador. 
Tabela 2- Comparação entre os dados obtidos da indústria e os resultados das simulações do HYSYS ${ }^{\circledR}$, para as temperaturas ao longo do DT.

\begin{tabular}{c|c|c|c}
\hline & \multicolumn{2}{|c|}{${\text { Temperatura }\left({ }^{\circ} \mathbf{C}\right)}$} \\
\hline Estágios & Real & HYSYS ${ }^{\circledR}$ & Desvio(\%) \\
\hline $\mathbf{1}$ & 71,00 & 63,67 & 10,3 \\
\hline $\mathbf{2}$ & 90,00 & 80,98 & 10,0 \\
\hline $\mathbf{3}$ & 100,00 & 109,60 & 9,6 \\
\hline $\mathbf{4}$ & 101,00 & 108,30 & 7,2 \\
\hline $\mathbf{5}$ & 108,00 & 107,10 & 0,8 \\
\hline $\mathbf{6}$ & 110,00 & 105,90 & 3,7 \\
\hline $\mathbf{7}$ & 115,00 & 104,70 & 9,0 \\
\hline
\end{tabular}

Por outro lado, uma análise da quantidade de hexano residual no farelo de soja, e da quantidade de hexano retirado nas etapas de dessolventização para diferentes vazões de vapor alimentadas, foi realizada com o intuito de avaliar a vazão mínima de vapor necessária para efetuar uma efetiva remoção do hexano a partir da torta. Desta forma, posteriormente será possível avaliar as melhores condições de operação do processo de forma a reduzir o consumo energético do processo de dessolventização/tostagem, que é crítico nas indústrias de processamento de soja. Tal análise foi realizada a partir de simulações utilizando o modelo desenvolvido para o dessolventizador. É possível observar nas Figuras 3 e 4 que, para baixas vazões de alimentação de vapor, ocorre uma queda considerável na retirada de hexano e consequentemente um aumento na vazão de hexano residual que tende a se acentuar ainda mais para vazões mais baixas, o que torna inviável a utilização de menores vazões na planta, tendo em vista a contaminação do meio ambiente, segurança industrial e qualidade do farelo de soja.

Figura 3-Vazão de hexano residual em função da vazão de vapor.

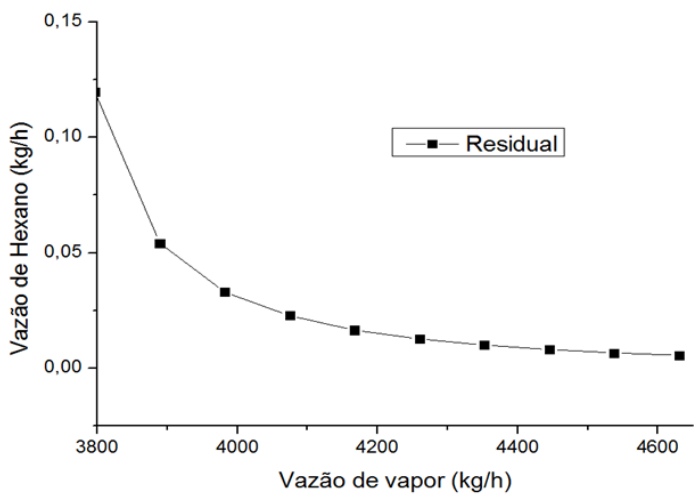

Figura 4-Vazão de hexano retirado em função da vazão de vapor.

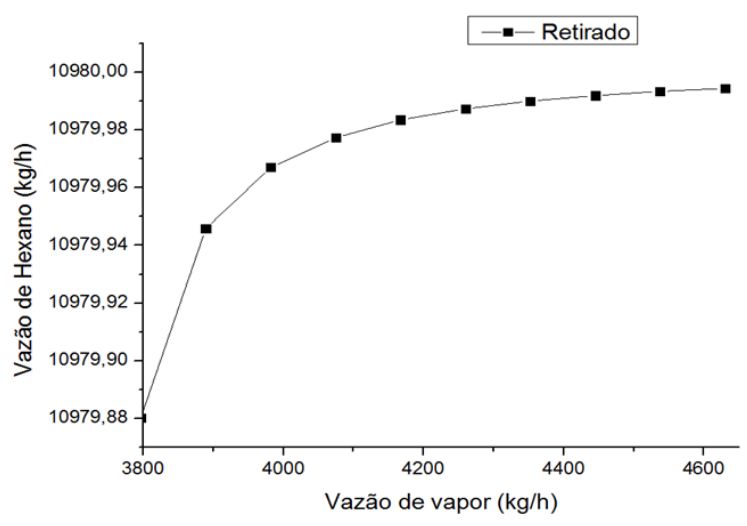




\section{CONCLUSÃO}

Os resultados obtidos por meio de simulações computacionais do modelo desenvolvido no presente trabalho para um dessolventizador/tostador de três estágios no tostador apresentaram desvios relativos baixos em relação aos valores encontrados na indústria de processamento de soja.

O modelo foi adaptado para cinco estágios no tostador e apresentou maiores desvios em relação à temperatura nos últimos estágios exibindo uma queda na temperatura durante a etapa de tostagem, o que não ocorre no processo real, sinalizando que o modelo de equilíbrio utilizado para realizar as simulações computacionais pode não ser adequado para representar os estágios de equilíbrio, que constituem os estágios do dessolventizador/tostador. Desta forma, outros pacotes de equilíbrio devem ser testados a fim de se obter novos dados numéricos para a comparação com o processo real e posterior estudo do processo de tostagem.

Já para o dessolventizador pôde-se realizar uma análise da quantidade de hexano retirada para diferentes vazões de vapor alimentadas e observou-se que para vazões de vapor inferiores a $3800 \mathrm{~kg} / \mathrm{h}$ o processo se torna inviável, tendo em vista a contaminação do meio ambiente, segurança industrial e qualidade do farelo de soja, devido à quantidade de hexano residual.

\section{REFERENCIAS}

CARDARELLI, D.A., \& CRAPISTE, G. H. (1996). Hexane sorption in oilseed meals. Journal of American Oil Chemists' Society, 73, 1657-1662.

DAHLEN, J.A.H., \& LINDH, L.A. (1983). Mass balance of hexane losses in a extraction plant. Journal of the American Oil Chemists' Society, 60, 2009-2010.

GRANT, D.R., EAGER, R. L., PEPPER, J. M., \& MATHEWS, J. F. (1983). Factor affecting the desolventization of canola meal. Journal of the American Oil Chemists' Society, 60, 18671875 .

KEMPER, G. T. Innovations in Meal Desolventizing, In: AMERICAN OILS CHEMISTS SOCIETY, 91, San Diego, 2000. Proceedings.AOCS, 2000.

MENEGUELO, A. P., Contribuições à análise e modelagens de operações transientes de colunas de destilação, Tese de Doutorado - DEQ/UFSC, 2007Schumacher, H. (1983). Ultimate energy possibilities in conventional solvent extraction. Journal of American Chemists' Society, 60, 417-419.

MUSTAKAS, G. C. et al., Critical Processing Factors in Desolventizing-Toasting Soybean Meal for Feed, JAOCS Journal of the American Oil Chemists Society, p. 300, March, 1981.

PARAÍSO, P. R., Modelagem e Análise do Processo de Obtenção do Óleo de Soja, Tese de Doutorado - FEQ/UNICAMP, 2001. 
PARAÍSO, P.R., Cauneto H., Zemp R.J., Andrade C.M.G., Modeling and simulation of the soybean oil meal desolventizing-toasting process. Journal of Food Engineerig, 86, 334-341.

PEGDEN, C. D,; SHANNON, R. E.; SADOWSKI, R. P. Introduction to simulation using SIMAN. McGraw-Hill, NY. 2 ed., 1990.

WOLFF, J. P. (1983). Residual hexane in meals. Journal of the American Oil Chemists' Society, 60, 220-223. 